\title{
Reexamination of the Requirements to Detect the Failure Wave Velocity in SiC Using Penetration Experiments
}

\author{
C. E. Anderson, Jr. ${ }^{1}$, D. L. Orphal ${ }^{2}$, D. W. Templeton ${ }^{3}$ \\ ${ }^{1}$ Southwest Research Institute, P. O. Drawer, San Antonio, TX 78240; \\ ${ }^{2}$ International Research Associates, 4450 Black Ave, Pleasanton, CA 94566; \\ ${ }^{3}$ U. S. Army TACOM-TARDEC, AMSTA-TR, Warren, MI 48397
}

Data for projectile penetration of silicon carbide (SiC) from two types of experiments were combined and analyzed in previous work [1-2]. Analysis of the data suggested the presence of the so-called "failure wave" phenomenon, that is, the apparent increase in the strength of SiC when the penetration velocity exceeds some critical value. These data are used as the basis for the design of a new set of experiments. The objectives of these new experiments are to remove ambiguities and uncertainties that exist in the analysis and interpretation of the original data sets and thereby more definitively detect and characterize the phenomena attributed to a "failure wave." The design requirements for experiments to achieve the high impact velocities necessary for investigating the physical phenomenon are described.

\section{Introduction}

Data from two data sets, long-rod tungsten projectiles and shaped-charge jets into $\mathrm{SiC}$ targets, were combined to examine the penetration resistance of $\mathrm{SiC}$ as a function of penetration velocity [1-2]. Analysis of the data suggested that there is an increase in the strength of the $\mathrm{SiC}$ above some threshold penetration velocity. Penetration velocity versus impact velocity is shown for the two data sets in Fig. 1. Also shown in the figure are the hydrodynamic limits$u_{\text {hydro }}=\mathrm{v} /\left(1+\sqrt{\rho_{t} / \rho_{p}}\right)$, where $u$ is the penetration velocity, $\mathrm{v}$ is the impact velocity, $\rho$ is the density, and the subscripts $t$ and $p$ indicate the target and projectile, respectively-for tungsten into $\mathrm{SiC}$ and copper into $\mathrm{SiC}$. It is noted that the tungsten into $\mathrm{SiC}$ data are approaching the hydrodynamic limit with increasing impact velocity, but that the copper into $\mathrm{SiC}$ data have the opposite trend.

An alternative representation of the data is shown in Fig. 2, where the difference in the hydrodynamic penetration velocity and the measured penetration velocity, normalized by the hydrodynamic penetration velocity, [( $\left.\left.u_{\text {hydro }}-u\right) / u_{\text {hydro }}\right]$, is plotted versus the impact velocity. Two curves are drawn through the data. The dotted curve is a exponential least-squares regression that extrapolates the tungsten data to higher velocities. The dashed line is a quadratic leastsquares regression fit to all the data. In this representation of the data, it is observed that the normalized difference in the penetration velocities begins to increase above an impact velocity of $\sim 5 \mathrm{~km} / \mathrm{s}$. 


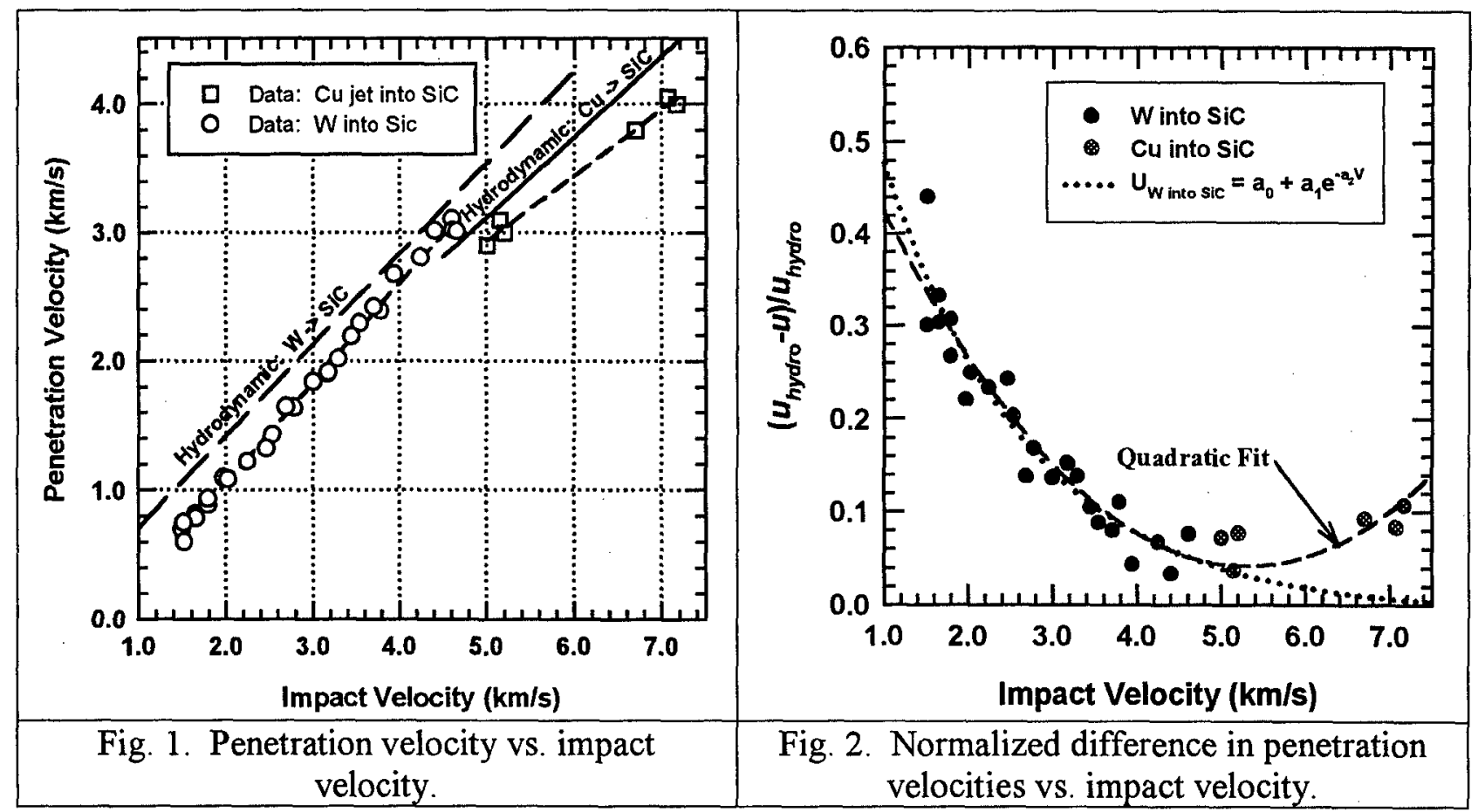

\section{Issues with Previous Experiments}

There are a number of issues with the previous experiments, which are discussed by the authors in Ref. [2]. These include, but are not necessarily limited to:

- The experiments used two different projectiles: a tungsten rod for the reverse ballistic tests and a portion of a copper shaped charge jet for the highest velocity experiments;

- Different pedigrees of silicon carbide (different processing and densities);

- The reverse ballistic tungsten rod tests used a semi-infinite target while the shaped charge jet experiments used a finite thickness ceramic target;

- Penetration velocity is inferred in the shaped charge experiments from arrival times at the front and back surface of the target;

- There is essentially no overlap in the experimental data sets.

\section{Design of New Experiments}

Higher Impact Velocity. Our analysis resulted in a decision to resolve the above issues associated with the previous research by conducting a new series of reverse ballistic experiments using a long rod penetrator. Based on the original analysis [1,2] it was concluded that impact velocities up to at least $6.5 \mathrm{~km} / \mathrm{s}$ are necessary to obtain a penetration velocity sufficiently high that the failure kinetics of the ceramic might be revealed with confidence.

New Target Design. The requirement for very high impact velocities meant that the targets had to have less mass than in the previous research. This required a redesign of the target to remove the titanium sleeve used previously, reduce both the diameter and length of the ceramic, remove the cover plate, and reduce the thickness of the back plate. A series of numerical simulations 
was performed to determine the minimum target diameter required to avoid effects of the radial boundary on penetration. For $\mathrm{SiC}$ the final target design had a ceramic diameter of $15-20 \mathrm{~mm}$ (which is $20-27$ projectile diameters) and a length of $40 \mathrm{~mm}$.

New SiC Material. The previous experiments used SiC-B. It was decided to use SiC-N for the new experiments since it is a ceramic of choice for armor applications. In order to be sure that the new experiments reproduced the previous results, a small number of experiments will be performed with both $\mathrm{SiC}-\mathrm{N}$ and $\mathrm{SiC}-\mathrm{B}$.

New Penetrator Material and Diameter. The previous research used a tungsten long rod of diameter, $D=0.762 \mathrm{~mm}$. The new experiments will use a gold rod with $D=0.75 \mathrm{~mm}$. Gold was selected for the penetrator material in order to have a very high density but very low strength penetrator. The low strength for the penetrator essentially removes effects of penetrator strength from analysis of the results.

Measurement Accuracy and Precision. Error analysis shows that resolving this expected transition requires higher accuracy measurements than were possible in the previous research. The accuracy and precision necessary are indicated in Fig. 3. The improvements and procedures implemented to achieve the necessary experimental accuracy are summarized in [3].

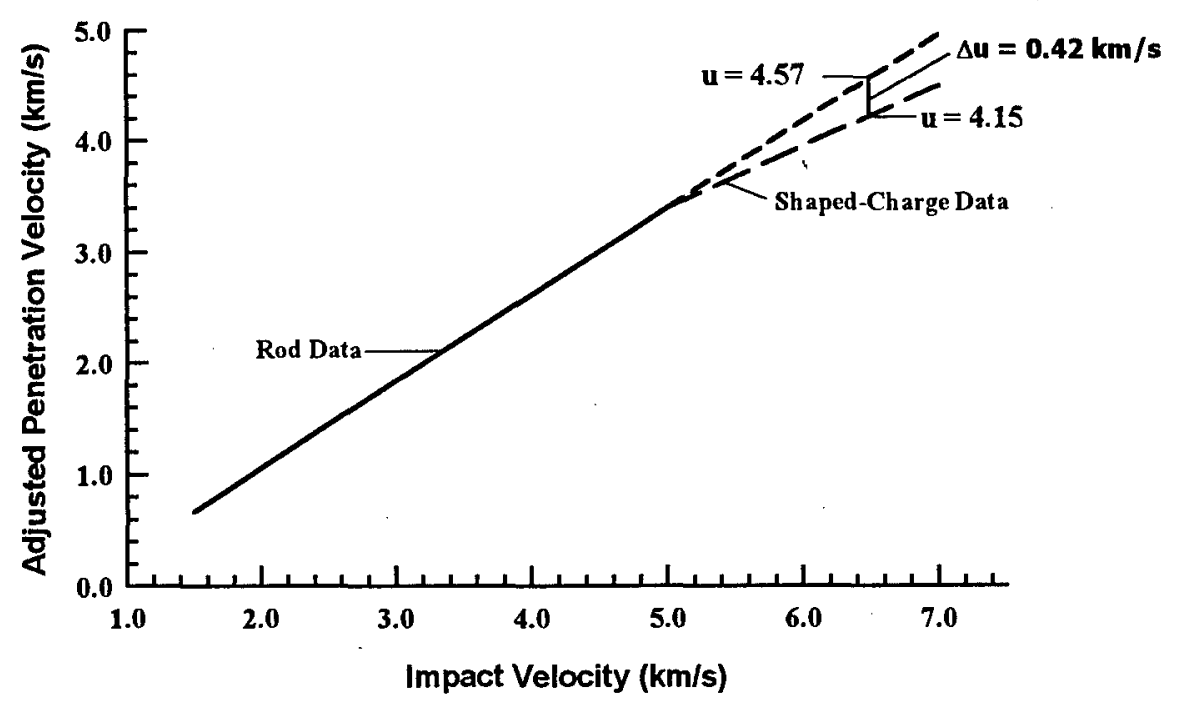

Testing. Initial tests have been successfully performed to validate the function and precision of the flash X-ray timing and analysis procedures. Testing is currently being conducted to demonstrate that the changes in the experiment design described above, as compared to the earlier work [1-2], have no unexpected effects on the penetration velocity.

\section{Hypothesis}

The increase in penetration resistance above some threshold penetration velocity has been attributed to the existence of a failure wave. For example, Partom postulates the existence of a failure wave for planar impact of glass [4]. Above some threshold stress, damage is assumed to 
propagate at a "failure wave" velocity that is pressure dependent. Behind the failure wave, there is a two to threefold reduction in the shear modulus. For penetration, the hypothesis is that failure propagates ahead of the projectile, so that the projectile penetrates failed material. If, however, the projectile penetrates the material faster than the propagating failure wave, then the projectile must penetrate undamaged, and therefore strong, material.

Although speculative at this point, we propose instead that the increase in penetration resistance is due to the kinetics of failure, that is, it takes a finite amount of time for material to undergo failure. If the projectile is penetrating with a velocity sufficiently high that full failure of the material directly in front of the projectile does not have time to occur, the material will respond as less than fully failed, and thus stronger, material.

A schematic of our current thinking regarding the failure mechanism is shown in Fig. 4 . A damage variable, $D^{*}$, represents failure, with " 0 " being no failure and " 1 " being complete failure. In this context, these rod penetration experiments can be interpreted as showing that below $u \sim 3.2 \mathrm{~km} / \mathrm{s}$, the material in front of the projectile has sufficient time to completely fail and the projectile is moving into totally failed (comminuted) material $\left(D^{*}=1\right)$. However, once failure is initiated, by whatever mechanism, there is an "incubation" time for "full" damage to be realized (dislocation motion, microcracking, coelescence, etc.). At sufficiently high velocities, e.g., the data in [1-2] would suggest $u$ greater than about $3.8 \mathrm{~km} / \mathrm{s}$, the projectile is penetrating material that has not had time to transition from intact to fully failed material; and consequently, the penetration resistance increases. An objective of the present work, in addition to the experimental efforts, is to investigate this hypothesis.

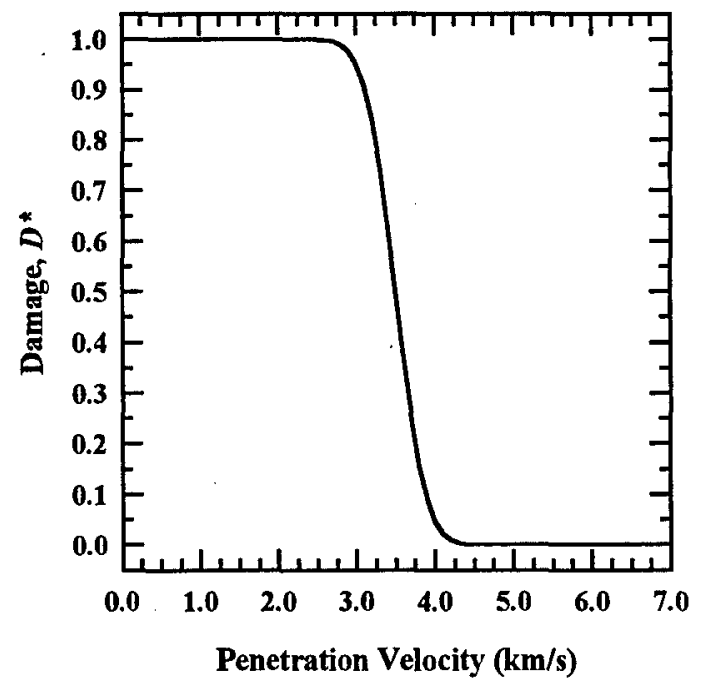

\section{References}

1. D. L. Orphal, A. A. Kozhushko, and A. B. Sinani, Shock Compression of Condensed Matter - 1999, Edited by M. D. Furnish, et al, pp. 577-580, AIP, 2000.

2. A.A. Kozhushko, D.L. Orphal, A.B. Sinani, and R.R. Franzen, Int. J. Impact Engng., 23, pp. 467-475, 1999. 
3. Th. Behner, V. Hohler, C. E. Anderson, Jr., and D.L. Orphal, $21^{\text {st }}$ Int. Symp. on Ballistics, Adelaide, Australia, submitted, April 2004.

4. 4.Y. Partom, Int. J. Impact Engng., 21(9), 791-799, 1998. 
16166

OPSEC REVIEW CERTIFICATION

(AR 530-1, Operations Security)

I am aware that there is foreign intelligence interest in open source publications. I have sufficient technical expertise in the subject matter of this paper to make a determination that the net benefit of this public release outweighs any potential damage.

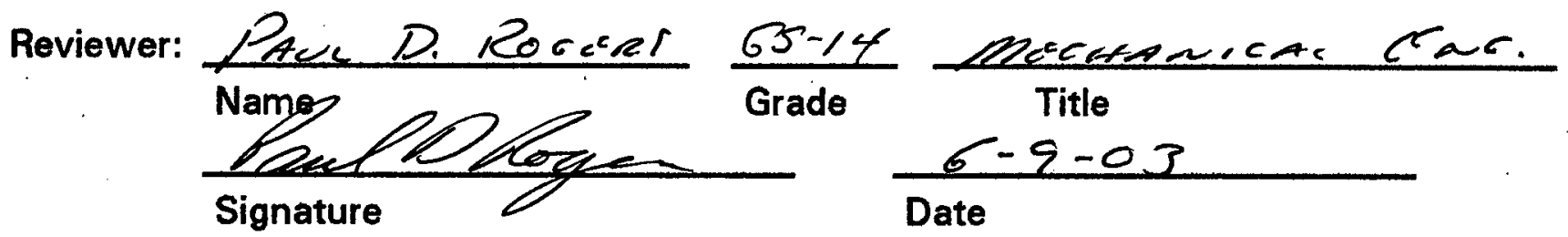

Description of Information Reviewed:

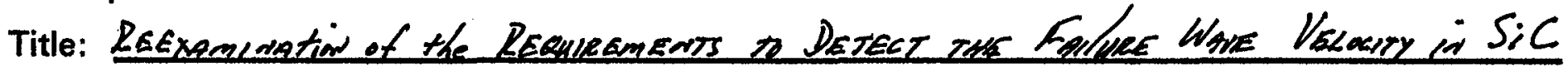
Author/Originator(s): C.E. Arsersad, D.L. Orphol, D. W. TEmpletor

Publication/Presentation/Release Date: AmERican Physical Society, $20.25 J_{U N} 03$

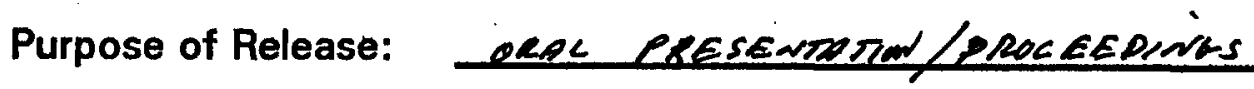

abstract, summary, or copy of the information reviewed is available for review.

Reviewer's Determination (check one)

(1.) Unclassified Unlimited.

2. Unclassified Limited, Dissemination Restrictions IAW

3. Classified. Cannot be released, and requires classification and control at the level

of

Security Office (AMSTA-CM-XS):

Concur/Nonconcur


STA 7114-E 